\title{
Enhanced Oxidative Desulfurization in a Film-shear Reactor
}

2

3

$4 \quad{ }^{\mathrm{a} D e p a r t m e n t}$ of Chemistry and Biochemistry, 1253 University of Oregon, Eugene, OR 97403

5
Brandy R. Fox, ${ }^{a}$ Benjamin L. Brinich, ${ }^{a}$ Jonathan L. Male, ${ }^{b}$ Robert L. Hubbard, ${ }^{c}$ Mohammad N. Siddiqui, ${ }^{\mathrm{d}}$ Tawfik A. Saleh, ${ }^{\mathrm{d}}$ and David R. Tyler ${ }^{a}$
${ }^{d}$ Department of Chemistry and Center of Research Excellence in Nanotechnology (CENT), King Fahd University of Petroleum \& Minerals, Dhahran 31261, Saudi Arabia
Corresponding author:
David R. Tyler
Department of Chemistry and Biochemistry
1253 University of Oregon
Eugene, OR 97403 USA
dtyler@uoregon.edu
$+1-541-346-4649$

7 
19 A film-shear reactor was used to significantly enhance the oxidative desulfurization (ODS) of

20 model fuels using hydrogen peroxide as the oxidant. Significant increases in the amount of

21 sulfur removed were seen in comparison to conventionally stirred ODS reactions. For example,

22 up to $50 \%$ desulfurization occurred in a single pass of the model fuel through the film-shear

23 reactor at $10{ }^{\circ} \mathrm{C}$. The desulfurization reactions were very fast in the reactor, occurring on the

24 time scale of seconds to minutes. Desulfurization was studied under a variety of conditions, and

25 a statistical design of experiment (DOE) showed that the fuel to oxidant ratio was the only

26 statistically significant parameter to impact the extent of desulfurization: a larger amount of

27 oxidant led to higher desulfurization. A variety of benzothiophene contaminants

28 (benzothiophene, 2-methylbenzothiophene, 5-methylbenzothiophene, dibenzothiophene, and 4,6-

29 dimethyldibenzothiophene) were examined, and the film-shear reactor was effective in removing

30 all of these contaminants. The film-shear reactor was effective at both low $(0.5-2.0 \mathrm{~mL} / \mathrm{min})$

31 and high $(100-300 \mathrm{~mL} / \mathrm{min})$ flow rates. Experiments showed that oxygen in air was not an

32 effective oxidant for ODS in the film-shear reactor. Experiments using $\mathrm{Mo}(\mathrm{CO})_{6}$ as a molecular

33 thermometer showed that "hot spots" are not forming in the film-shear reactor, and thus the

34 increase in the ODS rate cannot be attributed to intense thermal activation occurring within the

35 film-shear reactor. It is suggested that superb mixing of the aqueous and fuel phases is

36 responsible for the increased rate of ODS in the reactor.

38 Keywords Oxidative desulfurization, film-shear reactor, sulfur heterocycles, recalcitrant

39 thiophenes, biphasic reaction

40 


\section{Introduction}

42 The effect of high shear and superb mixing on reaction rates is an active area of process

43 intensification research [1-8]. One method of increasing shear and mixing is to use a film-shear

44 reactor (Figure 1) [9]. A film-shear reactor is a device with a rotating disk (the rotor) placed at

45 an adjustable distance $20-300 \mu \mathrm{m}$ from a stationary disk (the stator). The rotor spins at speeds up

46 to $10^{4} \mathrm{rpm}$, and two solution streams containing the reactants are introduced between the two

47 disks. Contact of the fluids within the narrow gap results in intense shear with consequent

48 intimate mixing of the two solutions.

Figure 1 here

In a previous communication [10], we reported significant enhancement of a biphasic

53 oxidative desulfurization (ODS) reaction using a film-shear reactor with hydrogen peroxide as

54 the oxidant. For example, up to $55 \%$ of the benzothiophene in a model fuel was oxidized in the

55 few seconds required for a single pass of the model fuel through the reactor at room temperature

56 or lower temperatures; further desulfurization was achieved when the model fuel was

57 recirculated through the reactor. In comparison, negligible oxidation of the benzothiophene

58 occurred under these conditions using conventional stirring. In this report, we expand on the use

59 of a film-shear reactor for ODS. Specifically, we extend the ODS process to thiophene

60 contaminants other than benzothiophene, and we investigate the effect of much higher flow rates

61 through the reactor on the efficiency of ODS. We also report the results of experiments using

62 molecular oxygen rather than hydrogen peroxide as the oxidant in the ODS process. Finally, we 
63 report the results of a mechanistic investigation into whether the film-shear reactor can thermally

64 activate molecules.

\section{Experimental}

For this investigation, a $0.5 \%$ solution of the indicated thiophene in decane was employed as a model fuel. A $5 \%$ solution of formic acid in $30 \% \mathrm{H}_{2} \mathrm{O}_{2}$ mixed with an equal volume of

69 isopropanol (to enhance sulfone solubility) was used as the oxidant stream. The aqueous and

70 decane phases separated readily upon exiting the reactor, facilitating quantitative analysis by ${ }^{1} \mathrm{H}$

71 NMR spectroscopy of the remaining sulfur in the decane layer. Specifically, analysis of the

72 signal intensity in the aromatic region relative to 1,1,2,2-tetrachloroethane as an internal standard

73 allowed calculation of the remaining thiophene (Figures S1-S5).

74 2.1. Reagents

75 Benzothiophene (BT; 99\%), 2-methylbenzothiophene (2-MeBT; 97\%), and 4,6-

76 dimethyldibenzothiophene (4,6-DMDBT; 97\%), dibenzothiophene sulfone (97\%) and $\gamma$ -

77 butyrolactone were obtained from Sigma-Aldrich. Dibenzothiophene (DBT; 98\%) was obtained

78 from Fluka. 5-methylbenzothiophene (5-MeBT; 98\%) and benzothiophene sulfone (98\%) were

79 obtained from Alfa Aesar. Decane (99\%) and1,1,2,2-tetrachloroethane were obtained from TCI.

80 Hydrogen peroxide (30-32\%) and isopropanol were obtained from Mallinckrodt. Deuterated

81 cyclohexane was obtained from Cambridge Isotope Laboratories. All reagents were used

82 without further purification.

83 2.2. Film-shear reactor trials

All film-shear reactor trials were carried out in a KinetiChem, Inc. Synthetron ${ }^{\mathrm{TM}}$ film-

85 shear reactor. The bulk temperature in the reaction zone was controlled using a recirculating 
86 temperature bath with a heat exchanger. The system was allowed to equilibrate at the indicated

87 temperature for 2 or more hours prior to setting the rotor-stator gap. Reagents were delivered

88 through Teflon tubing using independent SYR-2200 dual programmable syringe pumps obtained

89 from J-Kem Scientific. Upon reaching indicated reaction conditions, a minimum of 2 full

90 reaction volumes was allowed to flow through the reactor prior to sample collection in order to

91 ensure steady conditions.

92 For the desulfurization studies using $\mathrm{H}_{2} \mathrm{O}_{2}$, the fuel and oxidant feeds were introduced at

93 room temperature using independent syringe pumps. The feeds encountered each other in the

94 reaction zone (defined as the space within the rotor-stator gap). For the oxidation-by-air trials,

95 the $\gamma$-butyrolactone and model fuel phases were allowed to mix in a t-joint in the absence of air

96 prior to encountering the air in the reactor. Air flow was provided from a tank of Grade D

97 breathing air (19.5-23.5\% oxygen) and delivery was controlled using a flow meter.

\section{2.3. Sample preparation and analysis}

99 For both types of oxidation, the model fuel phase separated from the other phase

100 immediately upon exiting the reactor. $0.3 \mathrm{~mL}$ of the decane layer was combined in an NMR tube

101 with $0.3 \mathrm{~mL}$ deuterated cyclohexane containing $1,1,2,2-$ tetrachloroethane $(10-100 \mathrm{mM})$ as an

102 internal standard. Relative integrals of the aromatic region (compared to that of a sample of the

103 starting solution prepared in exactly the same way with the same internal standard solution) were

104 used to calculate the percent of the initial thiophene remaining. ${ }^{1} \mathrm{H}$ NMR spectra were recorded

105 a Varian 600 spectrometer at an operating frequency of $599.98 \mathrm{MHz}$.

106 For analysis of the $\gamma$-butyrolactone layer, $0.3 \mathrm{~mL}$ of the sample was combined in an NMR

107 tube with $0.3 \mathrm{~mL}$ deuterated methanol for locking. The ${ }^{1} \mathrm{H}$ NMR spectrum was obtained of this 
108 sample, which was then spiked with authentic thiophene. ${ }^{1} \mathrm{H}$ NMR spectra of the thiophenes in

$109 \gamma$-butyrolactone are shown in Figure S6.

110 2.4. Conventional mixing controls

111 For the control desulfurization studies (with $\mathrm{H}_{2} \mathrm{O}_{2}$ as the oxidant) using conventional

112 laboratory stirring, $10 \mathrm{~mL}$ of each reactor feed was placed in a $50 \mathrm{~mL}$ 2-neck round bottom flask

113 equipped with a magnetic stir bar and reflux condenser. The apparatus was placed in an oil bath

114 preheated to $80{ }^{\circ} \mathrm{C}$ and stirred vigorously. A sample $(1 \mathrm{~mL})$ was withdrawn from each phase at

115 the indicated time (in order to maintain equal volumes), and the decane layer was analyzed in the

116 manner reported for the reactor trials. (These results are illustrated in Figure 3 in the Results and

117 Discussion section.)

$118 \quad$ For desulfurization studies using air as the oxidant, $15 \mathrm{~mL}$ of each feed was placed in a 3-

119 neck round bottom flask equipped with a reflux condenser and stir bar. The second and third

120 necks were sealed with septa, and air was introduced into the solutions using a needle and

121 penetrating into the lower $\gamma$-butyrolactone phase. Air flow was maintained at $1 \mathrm{~mL} / \mathrm{min}$ using a

122 flow meter. This apparatus was placed in a preheated oil bath $\left(100{ }^{\circ} \mathrm{C}\right)$. At the indicated time, 1

$123 \mathrm{~mL}$ was withdrawn from each phase (in order to maintain equal volumes), and the decane and $\gamma$ -

124 butyrolactone layers were both analyzed in the manner described above for the film-shear reactor

125 trials. No oxidation was observed.

126 2.5. Checking for thermal "hot spots"

127 The reaction of $\mathrm{Mo}(\mathrm{CO})_{6}$ with $\mathrm{PPh}_{3}$ was used to check for thermal "hot spots." A recirculating

128 THF solution of $\mathrm{Mo}(\mathrm{CO})_{6}$ was reacted with a THF solution of $\mathrm{PPh}_{3}$ in the film-shear reactor at a

129 (bulk) temperature of $23^{\circ} \mathrm{C}$. One experiment was carried out for 5 hours and another for 33

130 hours. A strong absorption band in the infrared spectrum at $2072 \mathrm{~cm}^{-1}$ was used to quantify the 
131 amount of $\mathrm{Mo}(\mathrm{CO})_{5} \mathrm{PPh}_{3}$ that formed in the reaction system. For comparison, a conventionally

132 stirred reaction solution that did not pass through the reactor was put aside in the dark. For both

133 the 5-hour and the 33-hour experiments, the absorbances of the $2072 \mathrm{~cm}^{-1}$ band for the reaction

134 and control solutions was identical, indicating that an equal concentration of $\mathrm{Mo}(\mathrm{CO})_{5} \mathrm{PPh}_{3}$

135 formed in the two solutions.

137 3. Results and Discussion

138 3.1. Brief overview of oxidative desulfurization (ODS)

139 Oxidative desulfurization (ODS) [11-17] is a complementary alternative to current

140 hydrodesulfurization (HDS) technologies [18-22]. In the ODS reaction, an aqueous oxidant

141 (typically hydrogen peroxide with catalytic amounts of an acid) reacts with the sulfur

142 contaminant in the fuel phase. In current practice, ODS is used after HDS to remove sterically

143 hindered polycyclic aromatics, the so-called "recalcitrant thiophenes" [23-30]. The thiophene

144 contaminants are oxidized to sulfones (Scheme 1), which are easily removed from the fuel

145 because they are not soluble in the fuel phase [11-13]. ODS is appealing because the sulfur

146 contaminants that are most resistant to HDS, the recalcitrant thiophenes, are the most reactive

147 under ODS conditions [31-33]. However, because an aqueous oxidant (e.g., $\mathrm{H}_{2} \mathrm{O}_{2}$ ) must react

148 with the sulfur-containing contaminant in the fuel (organic) phase, hours of stirring at elevated

149 temperatures are generally required to carry out the ODS process. It was hypothesized that the

150 film-shear reactor could increase the rate of the biphasic ODS reaction by enhancing the mixing

151 of the fuel and aqueous phases. This was indeed the case, as we reported earlier for

152 benzothiophene [10]. 


\subsection{Extending the ODS process to contaminants other than benzothiophene}

157 Experiments were carried out to determine if the results with benzothiophene (BT) could 158 be extended to other benzothiophene contaminants. For this study, $0.5 \%$ dibenzothiophene 159 (DBT), 5-methylbenzothiophene (5-MeBT), 2-methylbenzothiophene (2-MeBT), 4,6160 dimethyldibenzothiophene (4,6-DMDBT), and BT were investigated (Figure 2). The model fuel

161 consisted of one of these thiophenes in decane, and the oxidant solution was $30 \% \mathrm{H}_{2} \mathrm{O}_{2}$, mixed

162 1:1 with isopropanol. The catalyst, formic acid, was included in the oxidant solution. The 163 purpose of the isopropanol was to prevent the sulfone from clogging the reactor because the 164 sulfone is poorly soluble in water [34]. To ensure a fair comparison of the results obtained with 165 the film-shear reactor to those obtained with conventional stirring, all of the control reactions 166 with conventional stirring also had isopropanol in the oxidant solution. Note that, among the 167 common organic solvents, acetonitrile would have been an excellent choice for extracting the 168 sulfone products [35]; however, control experiments showed that this solvent reacted in the film169 shear reactor; it was therefore unsuitable for these experiments.

Figure 2 here

174 syringe pumps. Upon exiting the reactor, the phases separated readily, and the thiophene

175 remaining in the decane layer was quantitatively analyzed by ${ }^{1} \mathrm{H}$ NMR relative to $1,1,2,2-$ 
176 tetrachloroethane as an internal standard. Sulfone products were identified by TLC by

177 comparison to authentic samples.

178 The results of the various trials are found in Table S1 and are summarized as follows:

179 The film-shear reactor can be used to carry out ODS of all the benzothiophenes shown in Figure

1802 ; in some cases, over $50 \%$ of the sulfur contaminant was removed with only short residence

181 times in the reactor (seconds to minutes, see Table S1). In contrast, the results for ODS by

182 conventional stirring are shown in Figure 3. In these experiments, desulfurization occurred on

183 the time scale of hours. Based on these comparisons, it is concluded that, compared to

184 conventional stirring, the film-shear reactor provides a significant enhancement of the ODS 185 process for a variety of benzothiophene contaminants.

Figure 3 here

\subsection{Factors affecting the efficiency of ODS in the film-shear reactor}

In order to elucidate the experimental and instrumental parameters that affect the extent

191 of desulfurization, a balanced DOE (statistical design of experiment) analysis was carried out

192 using BT as the sulfur-containing contaminant. (Details of the DOE analysis are found in the

193 Supporting Information.) The six parameters investigated in the DOE are shown in Table 1, and

194 the results are summarized as follows. Of the six parameters, only the fuel to oxidant ratio

195 ("ratio" in Table S2) is statistically significant. The effect of the ratio on the extent of sulfur

196 removal is logical: increasing the fuel to oxidant ratio results in less sulfur removal. Restated,

197 more oxidant leads to more oxidation of the BT and consequently more sulfur removal. 
201 3.4. Are the conditions for the ODS of benzothiophene also useful for other benzothiophenes?

202 As discussed above, if the film-shear reactor were ever to become practical for the ODS 203 process, it would have to remove a variety of benzothiophenes, not just BT, from fuels. It is 204 interesting to ask, therefore, if the reaction conditions that remove substantial amounts of BT will 205 also remove substantial amounts of the other benzothiophenes. To explore this question, the 206 oxidations of DBT, 5-MeBT, 2-MeBT, and 4,6-DMDBT were examined using the conditions 207 and parameters shown in Table 2. These conditions were selected for the trials because they 208 were among the best conditions for benzothiophene removal. Removal of the thiophenes was

209 monitored by ${ }^{1} \mathrm{H}$ NMR spectroscopy in the aromatic region, relative to 1,1,2,2-tetrachloroethane 210 as an internal standard. Representative spectra for each of the thiophene contaminants are found 211 in the Supporting Information, Figures S1-S5.

218 3), the results for some of the thiophenes were strikingly different from those of benzothiophene.

219 For example, there was essentially no desulfurization of 5-MeBT, 2-MeBT, and 4,6-DMDBT

220 under conditions A and B, which worked well for benzothiophene and to some extent for DBT.

221 This result was surprising because 5-MeBT, 2-MeBT, and 4,6-DMDBT were expected to be 222 more reactive than benzothiophene. [31-33,35] The third set of conditions (set C in Table 2)

223 was markedly better than the others, and provided especially effective desulfurization in the case 
224 of 4,6-DMDBT. A straightforward conclusion is that one set of conditions will probably not be

225 effective in removing all types of thiophenes. For practical applications, the best set of reaction

226 conditions would need to be determined for each contaminant.

227 It is not clear why a set of conditions that is so effective in removing one thiophene, say

228 benzothiophene under "condition A" or "condition B", is so ineffective at removing the other

229 thiophenes, especially when those thiophenes are expected to be more reactive than BT. These

230 results suggest that the function of the film-shear reactor is to provide more than superb mixing

231 and that some other experimental parameters are also affecting the reactivity. Studies delving

232 into what those other parameters might be are currently underway in our laboratory.

\section{3.5. High Combined Flow Rate}

235 In an attempt to increase the throughput of the ODS process in the film-shear reactor, a

236 set of experiments at high flow rates was conducted using benzothiophene as the contaminant.

237 For these experiments, the flow rate was varied between 100-300 $\mathrm{mL} / \mathrm{min}$, which represents an

238 increase of approximately 2 orders of magnitude over the experiments described previously. The

239 experimental results and the conditions are outlined in Table 4. Hydrogen peroxide and formic

240 acid concentrations were held constant for these experiments at $30 \%$ and $5 \%$, respectively.

241 Desulfurization ranged from 2 - 25\%, with values between $10-20 \%$ being typical. While these

242 yields are slightly lower than for the slower flow rates in Table S1 $(0.2-2.0 \mathrm{ml} / \mathrm{min})$, it is

243 interesting to point out that, at these high flow rates, the residence times range from 0.1-2.4 sec.

244 In addition, because it has already been shown that recirculation of the fuel results in further

245 desulfurization [10], one could imagine a set of film-shear reactors in series operating at these

246 high flow rates as a highly effective approach to desulfurization. 
Table 4 here

250 3.6. Using oxygen in air as the oxidant

251 In an attempt to eliminate the need for hydrogen peroxide, ODS was carried out in the

252 film-shear reactor using $\mathrm{O}_{2}$ as the oxidant. If successful, this desulfurization system would be

253 ideal for portable applications where access to hydrogen peroxide might be limited. These

254 attempts were based on the method of Makee, et al., who were able to effectively remove

255 thiophene contaminants from fuels using oxygen in air as an oxidant when $\gamma$-butyrolactone was

256 used as a cosolvent [36].

257 The necessary reagents were introduced into the reactor as illustrated in Figure 4, and the

258 sulfur remaining in the fuel layer after the reaction was quantified by ${ }^{1} \mathrm{H}$ NMR spectroscopy as in

259 the preceding experiments (Figure S6). Experiments were run at temperatures up to $80^{\circ} \mathrm{C}$. In

260 these experiments, up to $98 \%$ of the thiophenes were removed, but the removal was found to be

261 simply due to extraction of the thiophene into the $\gamma$-butyrolactone layer. (The presence and

262 identity of the thiophene in the $\gamma$-butyrolactone layer was confirmed by spiking the sample with a

263 sample of authentic thiophene for each trial.) Each of the thiophenes listed in Figure 2 was

264 examined in this manner, and no sulfone formation was observed under any of the experimental

265 conditions. The inability to use oxygen in air as an oxidant is consistent with the results of

266 control reactions using conventional stirring, which found that no detectable oxidation occurred

267 after stirring overnight at $100{ }^{\circ} \mathrm{C}$ in the presence of oxygen in air. It is noted that, in their

268 original report, Makee et al. reported that $140{ }^{\circ} \mathrm{C}$ was necessary for the ODS reaction to occur 
269 with oxygen in air. For technical reasons, this temperature was not achievable in the film-shear 270 reactor.

Figure 4 here

273

274 3.7. Does the film-shear reactor thermally activate molecules?

275 For biphasic reactions, it is generally assumed that superb mixing is one of the factors

276 responsible for the increased rates of reactivity in the film-shear reactor. Another possible

277 reason for the enhanced activity is that high shear rates may thermally activate molecules due to

278 the high frictional forces on the solvent molecules. Thermal activation could potentially arise

279 from either bulk warming of the reaction solution or from the presence of localized thermal "hot

280 spots." Bulk warming of the solutions was straightforwardly ruled out: our temperature

281 measurements of the reaction solution as it emerged from the reactor indicated only a slight

282 warming of the solution by several degrees, certainly not enough of a temperature increase to

283 cause an increase in the ODS rates by a factor of $10^{2}-10^{3}$ as reported here.

284 The possibility of localized thermal hot spots was probed by using molecules with well-

285 defined reaction rates as "molecular thermometers" [37,38]. Specifically, the substitution

286 reaction of the $\mathrm{Mo}(\mathrm{CO})_{6}$ complex by $\mathrm{PPh}_{3}$ (eq 1) was selected as a suitable molecular

287 thermometer because the rates and activation parameters for the reaction are well known $[39,40]$.

288 The procedure was to react $\mathrm{Mo}(\mathrm{CO})_{6}$ with $\mathrm{PPh}_{3}$ in the reactor (the metal complex in one feed

289 tube and $\mathrm{PPh}_{3}$ in the other) and to monitor the concentration of $\mathrm{Mo}(\mathrm{CO})_{6}$ and $\mathrm{Mo}(\mathrm{CO})_{5} \mathrm{PPh}_{3}$ as a

290 function of time as the reaction solution was repeatedly recirculated through the reactor. From

291 this data, the rate constant for the reaction can be obtained. Using the rate constant and the 
292 known values for $\Delta \mathrm{H}^{\dagger}$ and $\Delta \mathrm{S}^{\dagger}$, the Eyring equation can then be used to back-calculate the 293 effective temperature in the reaction zone.

equation 1 here

The reaction of $\mathrm{Mo}(\mathrm{CO})_{6}$ with $\mathrm{PPh}_{3}$ was carried out in the film-shear reactor for 5 hours

298 at a (bulk) temperature of $23{ }^{\circ} \mathrm{C}$. As a control, a conventionally stirred reaction solution that did

299 not pass through the reactor was put aside in the dark. Infrared spectroscopy was used to

300 quantify the amount of $\mathrm{Mo}(\mathrm{CO})_{5} \mathrm{PPh}_{3}$ that formed in each reaction system. (The product has a

301 strong absorption band at $2072 \mathrm{~cm}^{-1}[41,42]$.) The spectra showed that an equal concentration of

$302 \mathrm{Mo}(\mathrm{CO})_{5} \mathrm{PPh}_{3}$ formed in the two solutions. The reaction was repeated with a longer reaction

303 time (33 hours), but again the two solutions had equal concentrations of $\mathrm{Mo}(\mathrm{CO})_{5} \mathrm{PPh}$. From

304 these results, it is concluded that localized hotspots are not forming in the film-shear reactor.

\section{Summary and conclusions}

The film-shear reactor provides a significant enhancement of the ODS process compared

308 to conventional stirring for a variety of benzothiophene contaminants. A concern expressed by

309 some about our prior communication was that the flow rates of the fuel through the reactor were

310 quite small, certainly not large enough for any practical application. However, the work reported

311 here demonstrates that the increased efficiency of the ODS process in the reactor is maintained

312 even for flow rates as high as $300 \mathrm{~mL} / \mathrm{min}$. (At these flow rates, the residence times of the fuel

313 in the reactor are less than 1 second.) Another possible concern is that one would like to replace

$314 \mathrm{H}_{2} \mathrm{O}_{2}$ by oxygen in the air. As discussed, ODS reactions using oxygen in air as the oxidant have 
315 been reported at $140{ }^{\circ} \mathrm{C}$ using $\gamma$-butyrolactone as the solvent [36]. However, for technical

316 reasons this temperature is not feasible with the film-shear reactor, and no ODS occurred with

317 oxygen in air using the film-shear reactor at lower temperatures $\left(10-80^{\circ} \mathrm{C}\right)$. Experiments to

318 carry out the ODS process using oxygen rather than $\mathrm{H}_{2} \mathrm{O}_{2}$ are continuing in our laboratory.

319 Finally, the results reported here show that the film-shear reactor is not enhancing the rate of the

320 ODS process by thermally exciting the reactants in "hot spots" created by the high shear rates.

321 Superb mixing of the two immiscible phases is likely the primary reason for the increased rates,

322 but investigation of other reasons for the dramatically increased rates are also continuing in our

323 laboratory.

\section{Acknowledgments}

326 The authors acknowledge Dr. Jeffrey C. Raber at KinetiChem, Inc. who provided

327 substantial technical assistance and consultation. We are also grateful to B. Nell and A. Sun for

328 their experimental contributions. Initial funding for this research was provided by the National

329 Science Foundation (DGE-0231997) and the Army Research Laboratory (W911NF-07-2-0083).

330 For funding since 2014, the authors acknowledge the support provided by King Abdulaziz City

331 for Science and Technology (KACST) through the Science \& Technology Unit at King Fahd

332 University of Petroleum \& Minerals (KFUPM) through project No. 12-PET3009-04 as part of

333 the National Science, Technology and Innovation Plan (NSTIP).

\section{Appendix A. Supplementary Data}

336 Supplementary data associated with this article can be found, in the online version, at doi: 
338 [1] Rubin AE, Tummala S, Both DA, Wang C, Delaney EJ. Emerging technologies supporting

339 chemical process $\mathrm{R} \& \mathrm{D}$ and their increasing impact on productivity in the pharmaceutical

340 industry. Chem Rev Wash DC U S 2006;106:2794-810.

341 [2] Centi G, Perathoner S. Methods and tools of sustainable industrial chemistry: process

342 intensification., Wiley-VCH Verlag GmbH \& Co. KGaA; 2009, p. 199-255.

343 [3] Pohar A, Plazl I. Process intensification through microreactor application. Chem Biochem $344 \quad$ Eng Q 2009;23:537-44.

345 [4] Green A, Editor. Proceedings of the 3rd International Conference on Process Intensification 346 for the Chemical Industry: Smaller, Cheaper, and Safer Production, held 25-27 October 347 1999, in Antwerp, Belgium. [In: BHR Group Conf. Ser. Publ., 1999; 38]. Professional $348 \quad$ Engineering Publ Ltd.; 1999.

349 [5] Stankiewicz A, Drinkenburg AAH. Process intensification: history, philosophy, principles. $350 \quad$ Chem Ind Dekker 2004;98:1-32.

351 [6] Kumar V, Nigam KDP. Process intensification in green synthesis. Green Process Synth $352 \quad 2012 ; 1: 79-107$.

353 [7] Ghanem A, Lemenand T, Della Valle D, Peerhossaini H. Static mixers: Mechanisms, 354 applications, and characterization methods - A review. Chem Eng Res Des 2014;92:205-28.

355 [8] Boodhoo K. Spinning disc reactor for green processing and synthesis. Process Intensif 356 Green Chem, John Wiley \& Sons Ltd.; 2013, p. 59-90.

357 [9] Holl R. Reactor design: Adding some spin. Tce Chem Eng 2003;742:32-4.

358 [10] Fox BR, Sun AW, Dauer HB, Male JL, Stewart ML, Tyler DR. Enhanced oxidative 359 desulfurization of model fuels using a film-shear reactor. Fuel 2011;90:898-901. 
[11] Chica A, Gatti G, Moden B, Marchese L, Iglesia E. Selective catalytic oxidation of 361 organosulfur compounds with tert-butyl hydroperoxide. Chem - Eur J 2006;12:1960-7.

362 [12] Kong L, Li G, Wang X. Kinetics and mechanism of liquid-phase oxidation of thiophene 363 over TS-1 using H2O2 under mild conditions. Catal Lett 2004;92:163-7.

364 [13] Zhao D, Wang J, Zhou E. Oxidative desulfurization of diesel fuel using a Bronsted acid 365 room temperature ionic liquid in the presence of H2O2. Green Chem 2007;9:1219-22.

366 [14] Javadli R, Klerk A. Desulfurization of heavy oil. Appl Petrochem Res 2012;1:3-19.

367 [15] Garcia-Gutierrez JL, Lozano IP, Hernandez-Perez F, Laredo GC, Jimenez-Cruz F. R \& 368 D in oxidative desulfurization of fuels technologies: from chemistry to patents. Recent Pat 369 Chem Eng 2012;5:174-96.

370 [16] Chandra Srivastava V. An evaluation of desulfurization technologies for sulfur removal 371 from liquid fuels. RSC Adv 2012;2:759-83.

372 [17] Ismagilov Z, Yashnik S, Kerzhentsev M, Parmon V, Bourane A, Al-Shahrani FM, et al. 373 Oxidative Desulfurization of Hydrocarbon Fuels. Catal Rev Sci Eng 2011;53:199-255.

374 [18] Choudhary TV, Malandra J, Green J, Parrott S, Johnson B. Towards clean fuels:

375 molecular-level sulfur reactivity in heavy oils. Angew Chem Int Ed 2006;45:3299-303.

376 [19] Nova A, Novio F, Gonzalez-Duarte P, Lledos A, Mas-Balleste R. C-S bond activation 377 and partial hydrogenation of thiophene by a dinuclear trihydride platinum complex. Eur J $378 \quad$ Inorg Chem 2007:5707-19.

379 [20] Babich IV, Moulijn JA. Science and technology of novel processes for deep 380 desulfurization of oil refinery streams: a review. Fuel 2003;82:607-31.

381 [21] Girgis MJ, Gates BC. Reactivities, reaction networks, and kinetics in high-pressure 382 catalytic hydroprocessing. Ind Eng Chem Res 1991;30:2021-58. 
383 [22] Song C, Reddy KM. Mesoporous molecular sieve MCM-41 supported Co-Mo catalyst 384 for hydrodesulfurization of dibenzothiophene in distillate fuels. Appl Catal Gen 1999;176:1$385 \quad 10$.

386 [23] Velu S, Ma X, Song C. Selective Adsorption for Removing Sulfur from Jet Fuel over 387 Zeolite-Based Adsorbents. Ind Eng Chem Res 2003;42:5293-304.

388 [24] Liu D, Gui J, Song L, Zhang X, Sun Z. Deep Desulfurization of Diesel Fuel by 389 Extraction with Task-Specific Ionic Liquids. Pet Sci Technol 2008;26:973-82.

390 [25] Wang H, Prins R. Hydrodesulfurization of dibenzothiophene and its hydrogenated 391 intermediates over sulfided Mo/ $\gamma$-Al2O3. J Catal 2008;258:153-64.

392 [26] Song C. An overview of new approaches to deep desulfurization for ultra-clean gasoline, 393 diesel fuel and jet fuel. Catal Today 2003;86:211-63.

394 [27] Knudsen KG, Cooper BH, Topsoe H. Catalyst and process technologies for ultra-low 395 sulfur diesel fuel. Appl Catal Gen 1999;189:205-15.

396 [28] Wang J, Zhao D, Li K. Oxidative desulfurization of dibenzothiophene using ozone and 397 hydrogen peroxide in ionic liquid. Energy Fuels 2010;24:2527-9.

398 [29] Gates BC, Topsoe H. Reactivities in deep catalytic hydrodesulfurization: challenges, 399 opportunities, and the importance of 4-methyldibenzothiophene and 4,6400 dimethyldibenzothiophene. Polyhedron 1997;16:3213-7.

401 [30] Ma X, Sakanishi K, Mochida I. Hydrodesulfurization reactivities of various sulfur 402 compounds in diesel fuel. Ind Eng Chem Res 1994;33:218-22.

403 [31] Qian EW. Development of novel nonhydrogenation desulfurization process: oxidative 404 desulfurization of distillate. J Jpn Pet Inst 2008;51:14-31. 
405 [32] Sundararaman R, Ma X, Song C. Oxidative Desulfurization of Jet and Diesel Fuels

406 Using Hydroperoxide Generated in Situ by Catalytic Air Oxidation. Ind Eng Chem Res

$407 \quad 2010 ; 49: 5561-8$.

408 [33] Jiang X, Li H, Zhu W, He L, Shu H, Lu J. Deep desulfurization of fuels catalyzed by

409 surfactant-type decatungstates using H2O2 as oxidant. Fuel 2009;88:431-6.

410 [34] Hulea V, Fajula F, Bousquet J. Mild Oxidation with H2O2 over Ti-Containing

411 Molecular Sieves-A very Efficient Method for Removing Aromatic Sulfur Compounds from

$412 \quad$ Fuels. J Catal 2001;198:179-86.

413 [35] Otsuki S, Nonaka T, Takashima N, Qian W, Ishihara A, Imai T, et al. Oxidative

414 desulfurization of light gas oil and vacuum gas oil by oxidation and solvent extraction.

$415 \quad$ Energy Fuels 2000;14:1232-9.

416 [36] Xu X, Moulijn JA, Ito E, Wagemans R, Makkee M. Deep desulfurization of fossil fuels

417 by air in the absence of a catalyst. ChemSusChem 2008;1:817-9.

418 [37] Suslick KS, Schubert PF, Goodale JW. Sonochemistry and sonocatalysis of iron

419 carbonyls. J Am Chem Soc 1981;103:7342-4.

420 [38] Suslick KS, Gawienowski JJ, Schubert PF, Wang HH. Alkane sonochemistry. J Phys

$421 \quad$ Chem 1983;87:2299-301.

422 [39] Graham JR, Angelici RJ. Kinetic studies of Group VI metal carbonyl complexes. VI.

423 Substitution reactions of the Group VI metal hexacarbonyls. Inorg Chem 1967;6:2082-5.

424 [40] Werner H, Prinz R. Kinetic investigations of substitution reactions on metal complexes.

425 III. Mechanism of thermal reactions of metal hexacarbonyl compounds of chromium,

426 molybdenum, and tungsten with n-donors. Chem Ber 1966;99:3582-92. 
427 [41] Guns MF, Claeys EG, Van der Kelen GP. Infrared and carbon-13 NMR study of 428 phosphine monosubstituted group VIB metal carbonyls. J Mol Struct 1979;53:45-53.

429 [42] Hui K-Y, Shaw BL. Phase-transfer catalyzed substitution of Group VI metal carbonyls. J $430 \quad$ Organomet Chem 1977;124:262-4.

431 
434 Table 1. Parameters included in the DOE and the range over which they were surveyed. ${ }^{\mathrm{a}}$ The

435 voltage is used to adjust the rpm of the rotor. ${ }^{b}$ Refers to the volumetric ratio of the fuel phase to 436 the oxidant phase. ${ }^{\mathrm{c}}$ Reported as the percent formic acid in hydrogen peroxide used for the 437 oxidant phase ${ }^{\mathrm{d}}$ Flow rate of the 2 phases combined.

438 Table 2. The three sets of experimental conditions used to probe the oxidation of the thiophenes.

439 All of the trials used 5\% formic acid in $30 \% \mathrm{H}_{2} \mathrm{O}_{2}(1: 1$ with $i-\mathrm{PrOH})$.

440 Table 3. Extent of thiophene removal under the three conditions detailed in Table 2.

441 Table 4. High flow rate experiments and the extent of desulfurization.

443 Figure 1. Diagram of the film-shear reactor (not to scale).

444 Figure 2. The five thiophenes examined in this study

445 Figure 3. The fraction of thiophene remaining as a function of time during ODS carried out with 446 conventional stirring. Note that the desulfurization occurred on the time scale of hours.

447 Figure 4. Schematic of the reactor setup for oxidation by oxygen in the air. Air delivery was 448 controlled by way of a flow meter, and independent syringe pumps delivered the liquid 449 components to a t-joint for mixing in the absence of oxygen prior to entry into the reactor. 
Table 1. Parameters included in the DOE and the range over which they were surveyed. ${ }^{\mathrm{a}}$ The voltage is used to adjust the rpm of the rotor. ${ }^{b}$ Refers to the volumetric ratio of the fuel phase to the oxidant phase. ${ }^{c}$ Reported as the percent formic acid in hydrogen peroxide used for the oxidant phase ${ }^{\mathrm{d}}$ Flow rate of the 2 phases combined.

\begin{tabular}{lcc}
\hline \multicolumn{1}{c}{ Parameter } & Low Value & High Value \\
\hline Temperature $\left({ }^{\circ} \mathrm{C}\right)$ & 10 & 70 \\
Gap $(\mu \mathrm{m})$ & 250 & 1500 \\
Voltage $^{\mathrm{a}}(\mathrm{V})$ & 4 & 16 \\
Ratio $^{\mathrm{b}}$ & 1 & 10 \\
Composition $^{\mathrm{c}}(\%$ formic acid $)$ & 2.5 & 5 \\
Combined Flow Rate $^{\mathrm{d}}(\mathrm{mL} / \mathrm{min})$ & 0.5 & 2 \\
\hline
\end{tabular}


Table 2. The three sets of experimental conditions used to probe the oxidation of the thiophenes. All of the trials used $5 \%$ formic acid in $30 \% \mathrm{H}_{2} \mathrm{O}_{2}(1: 1$ with $i-\mathrm{PrOH})$.

\begin{tabular}{l|ccc}
\multirow{2}{*}{\multicolumn{1}{c}{ Parameter }} & \multicolumn{3}{c}{ Reaction Conditions } \\
\cline { 2 - 4 } & A & B & C \\
\hline Gap size (um) & 250 & 1500 & 250 \\
Temperature $\left({ }^{\circ} \mathrm{C}\right)$ & 10 & 70 & 70 \\
Ratio (fuel:ox) & 10 & 1 & 1 \\
CFR (mL/min) & 0.5 & 2 & 0.5 \\
Average RPM & 6300 & 1500 & 1500 \\
Residence time (sec) & 80 & 120 & 80 \\
Average max shear rate $\left(\mathrm{sec}^{-1}\right)$ & 84000 & 3500 & 19000
\end{tabular}


Table 3. Extent of thiophene removal under the three conditions detailed in Table 2.

\begin{tabular}{l|ccc}
\multirow{2}{*}{\multicolumn{1}{c}{ Thiophene }} & \multicolumn{3}{c}{ \% Thiophene Removal } \\
\cline { 2 - 4 } \multicolumn{1}{c}{ Benzothiophene } & A & B & C \\
\hline Dibenzothiophene & 44 & 51 & 55 \\
5-Methylbenzothiophene & 7 & 17 & 27 \\
5-Methylbenzothiophene (series B) & 0 & 0 & 13 \\
2-Methylbenzothiophene & 0 & 0 & 21 \\
4,6-Dimethylbenzothiophene & 0 & 0 & 13 \\
& 0 & 0 & 45
\end{tabular}


Table 4. High flow rate experiments and the extent of desulfurization.

\begin{tabular}{ccccc}
\hline $\begin{array}{c}\text { Combined } \\
\text { Flow Rate } \\
(\mathrm{mL} / \mathrm{min})\end{array}$ & $\begin{array}{c}\text { Rotation } \\
\text { Speed } \\
(\mathrm{RPM})\end{array}$ & $\begin{array}{c}\text { Gap } \\
(\mu \mathrm{m})\end{array}$ & $\begin{array}{c}\text { Temp. } \\
\left({ }^{\circ} \mathrm{C}\right)\end{array}$ & $\begin{array}{c}\text { Percent Sulfur } \\
\text { Removed }\end{array}$ \\
\hline 300 & 6300 & 250 & 70 & 15 \\
300 & 6300 & 250 & 10 & 2 \\
300 & 1600 & 1500 & 10 & 3 \\
300 & 1600 & 250 & 10 & 16 \\
100 & 6300 & 250 & 10 & 7 \\
100 & 1600 & 1500 & 70 & 13 \\
100 & 6398 & 1500 & 70 & 11 \\
200 & 3965 & 875 & 40 & 14 \\
100 & 1586 & 250 & 10 & 12 \\
300 & 6300 & 1500 & 70 & 18 \\
100 & 1537 & 1500 & 10 & 11 \\
100 & 6344 & 250 & 70 & 16 \\
100 & 6260 & 1500 & 10 & 15 \\
200 & 3919 & 875 & 40 & 15 \\
300 & 6300 & 1500 & 10 & 16 \\
300 & 1600 & 250 & 70 & 19 \\
300 & 1600 & 1500 & 70 & 29 \\
200 & 4031 & 875 & 40 & 24 \\
100 & 1600 & 250 & 70 & 25 \\
200 & 3930 & 875 & 40 & 19 \\
\hline & & & & \\
\hline
\end{tabular}


= heat exchanger

\section{Motor}

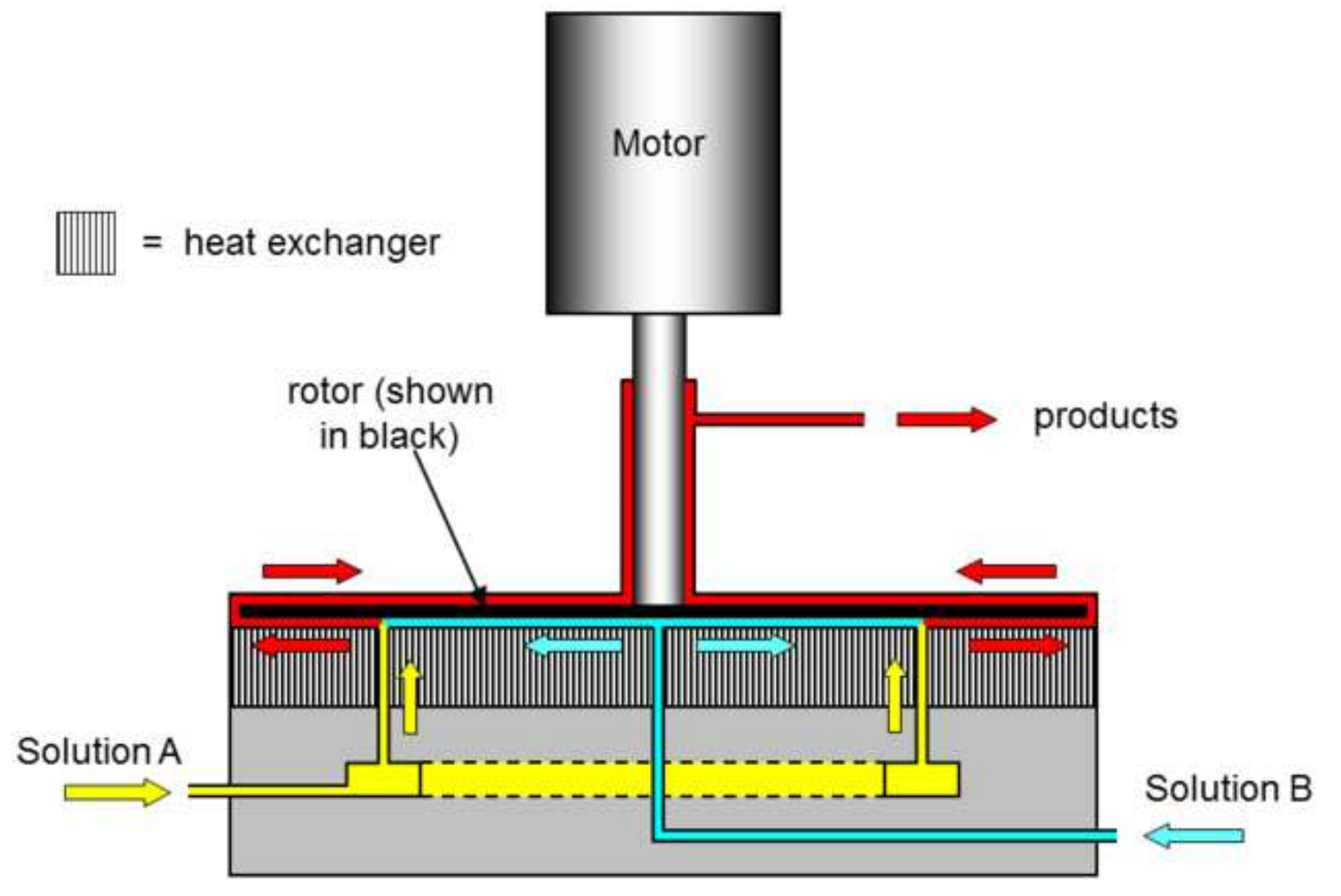

Solution B

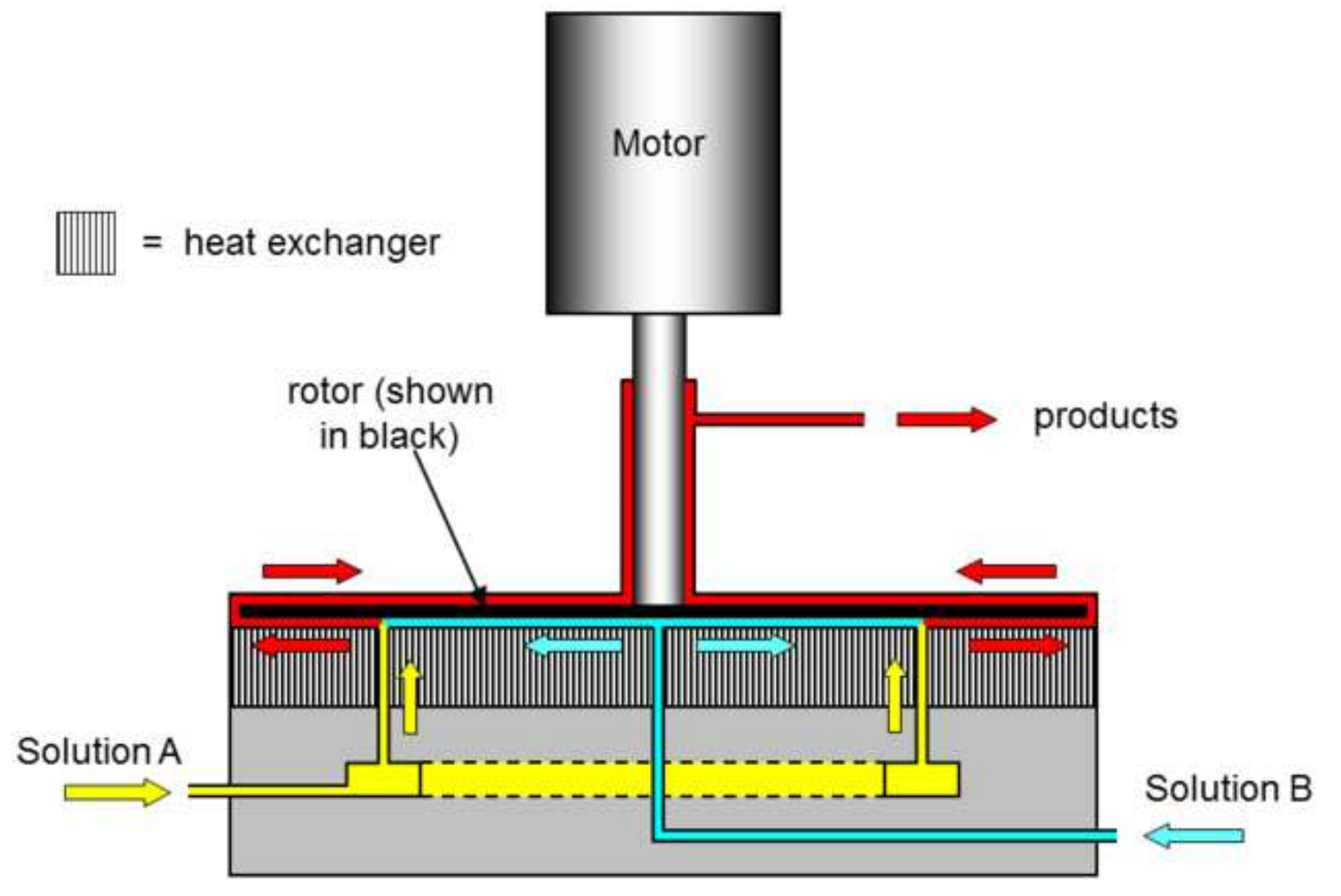




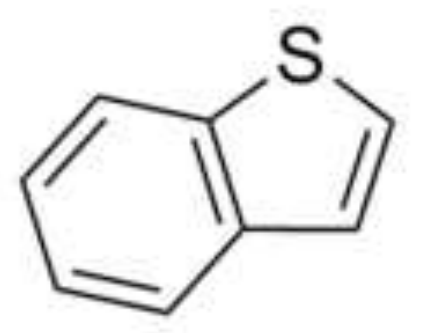

benzothiophene

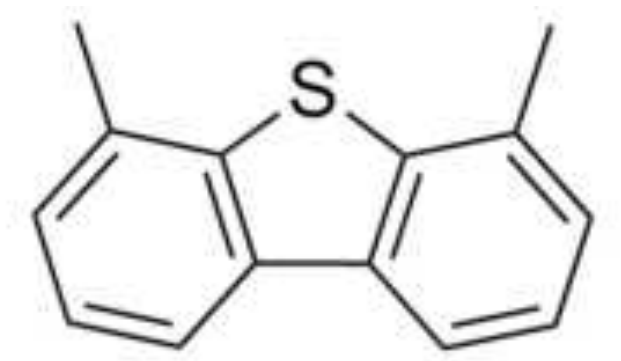

4,6-dimethyldibenzothiophene

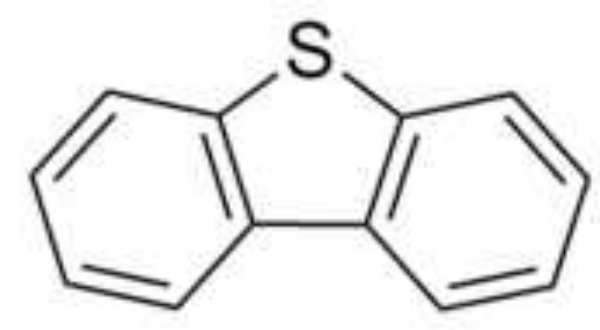

dibenzothiophene

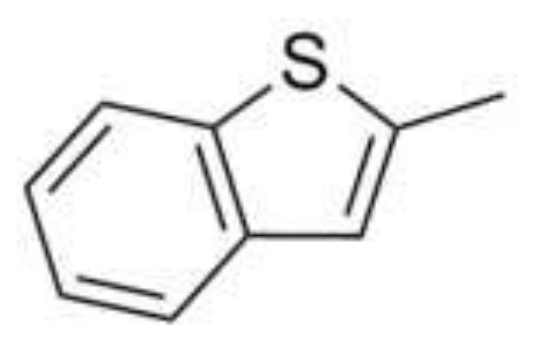

2-methylbenzothiophene

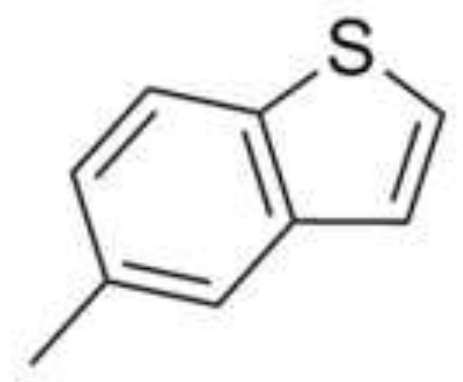

5-methylbenzothiophene 


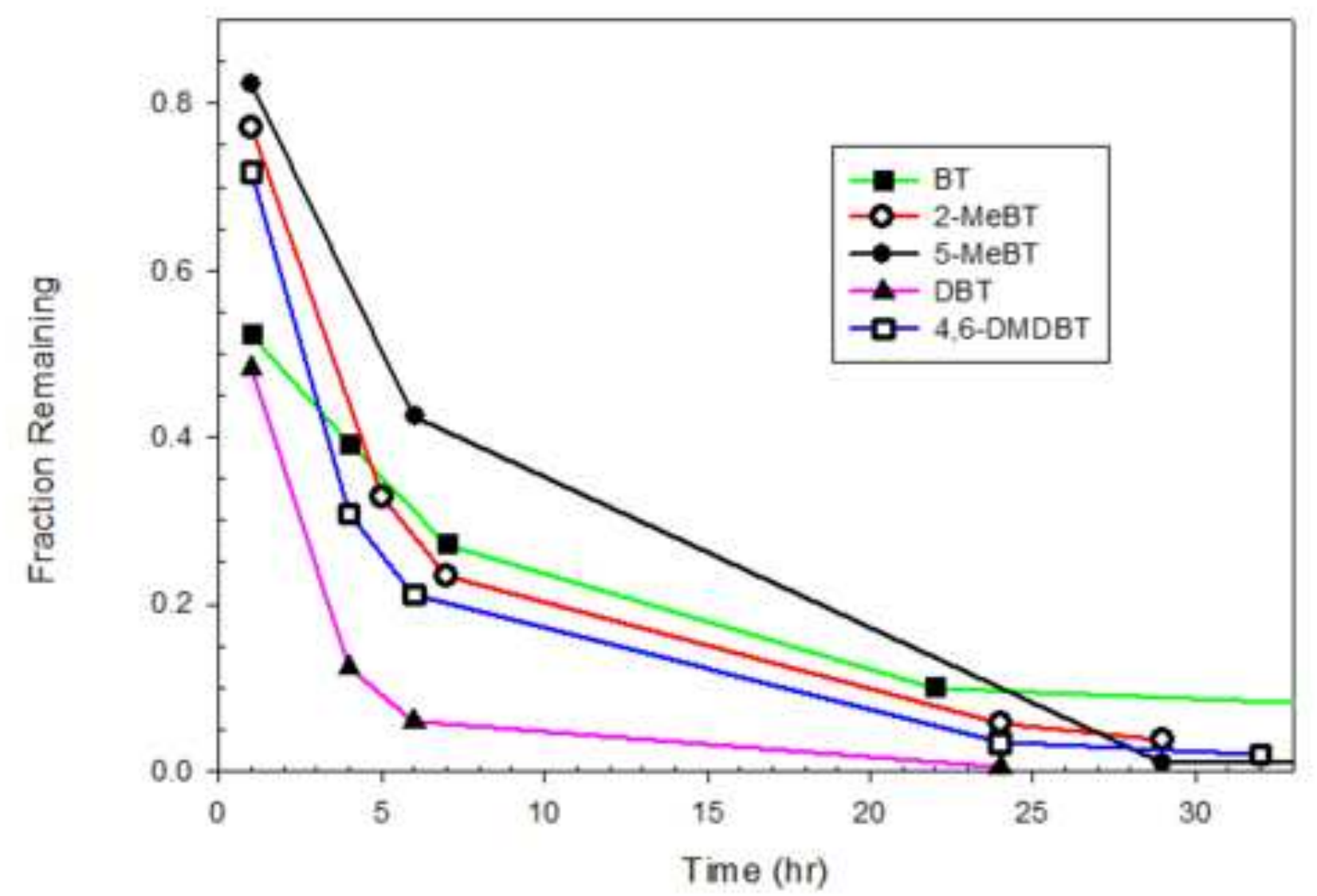




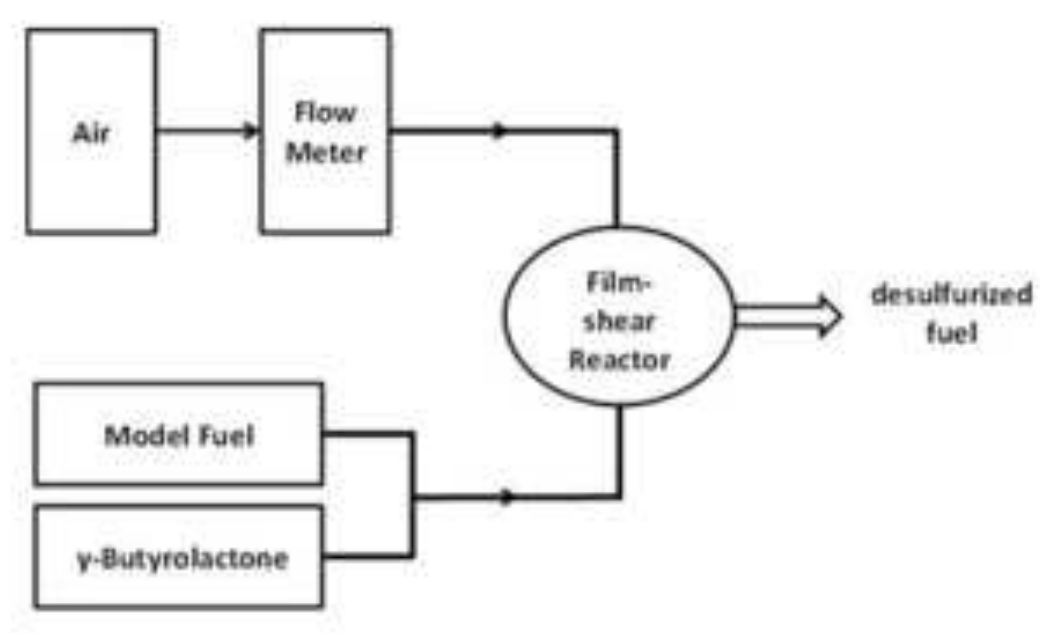


$\mathrm{Mo}(\mathrm{CO})_{6}+\mathrm{PPh}_{3}$ $\mathrm{Mo}(\mathrm{CO})_{5} \mathrm{PPh}_{3}+\mathrm{CO}$

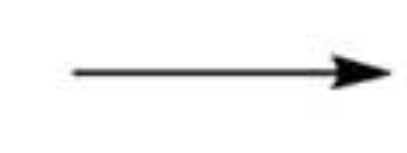

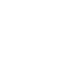

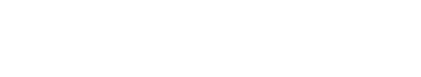

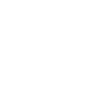

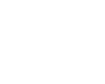

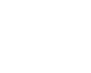


thiophene

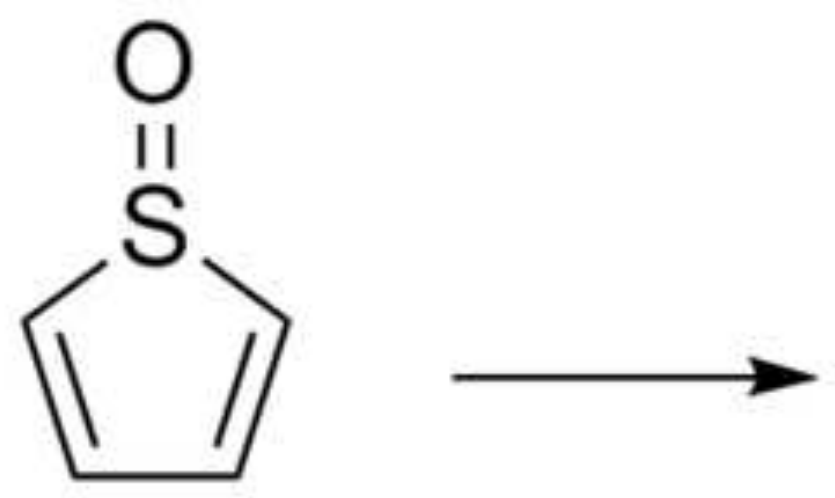

sulfoxide

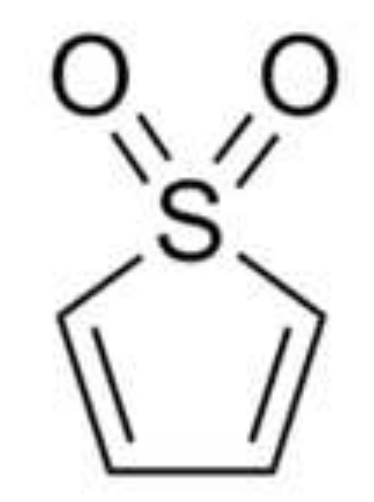

sulfone

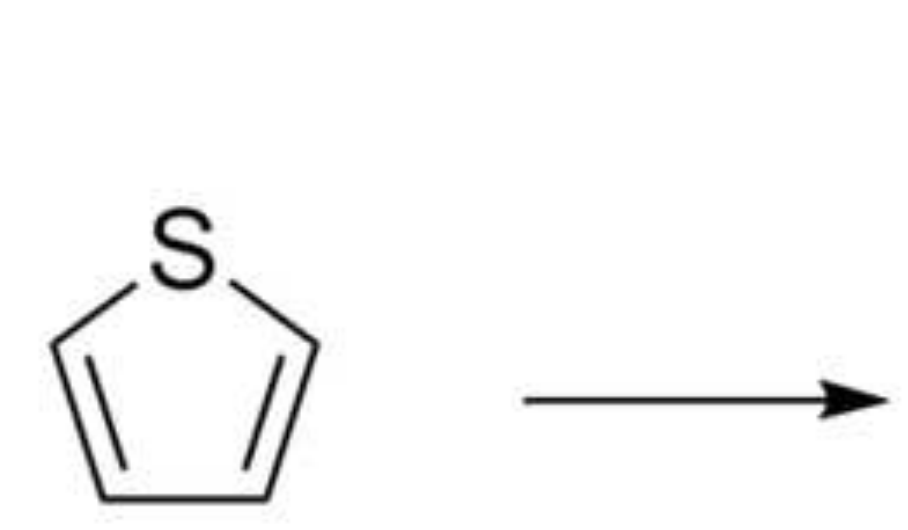

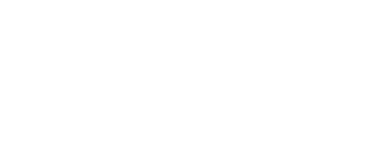


Graphical abstract

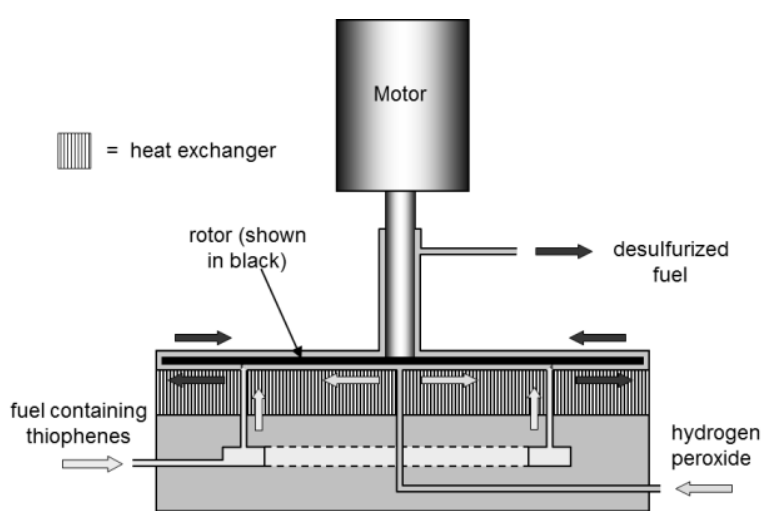

